\title{
Elektrochemische Synthesen, II $^{1}$ Die elektrochemische Reduktion von chemisorbiertem CO
}

\author{
Electrochemical Syntheses, $\mathrm{II}^{1}$ \\ The Electrochemical Reduction of Chemisorbed CO \\ Georg Bockmair und Heinz P. Fritz \\ Anorganisch-chemisches Institut der Technischen Universität München \\ (Z. Naturforsch. 30 b, 330-333 [1975]; eingegangen am 7. März 1975) \\ Electrochemical Reduction, Carbon-monoxide
}

\begin{abstract}
It was shown by cyclovoltammetry that carbon-monoxide dissolved in organic electrolyte can be reduced irreversibly at Ni-, Co-, and Ti-electrodes. A dependance of the potential on the metal was evident, corresponding to chemisorption and subsequent reduction of CO.
\end{abstract}

\section{Einleitung}

Die Möglichkeit Kohlenmonoxid zu reduzieren wurde bereits frühzeitig erkannt, so gelang in flüssigem Ammoniak die Synthese des Acetylendiolat-Dianions $(\mathbf{1})^{2}$ und des sechswertigen Anions des Hexahydroxobenzols $(\mathbf{2})^{3}$.
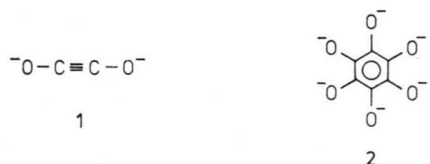

Spätere Arbeiten galten der Struktur des „Kaliumcarbonyls" alkalicarbonylen" der Summenformel $\mathrm{M}^{\mathrm{II}}(\mathrm{CO})_{2}{ }^{5}$. Bei der elektrochemischen Reduktion von Kohlenmonoxid (von $100 \mathrm{~atm}$ Druck) an Bleikathoden in neutraler Salzlösung konnte Methanol in gerade noch nachweisbaren Mengen gefunden werden ${ }^{6}$.

Diese Untersuchung läßt wegen der gewählten Versuchsanordnung, einer Elektrolyse bei konstantem Strom, keinen Schluß auf ein eventuell intermediär vorhandenes $\mathrm{CO}^{-}$-Ion zu. 1972 berichteten dann Silvestri et al. über die elektrochemische Reduktion von Kohlenmonoxid an Stahlkathoden

Sonderdruckanforderungen an Prof. Dr. H. P. Fritz, Anorg.-chem. Institut der Techn. Universität, D-8000 München 2, Arcisstraße 21. unter erhöhtem Druck zum Anion der Quadratsäure $(3)^{\text {? }}$.<smiles>OC1C(O)C(O)C1O</smiles>

Es fällt auf, daß offenbar bevorzugt eine gerade Zahl von CO-Spezies sich zusammenlagern zu 1, 2 oder 3 .

Eine ,,reduktive Carbonylierung“" als Synthese von Metallcarbonylen gelingt bekanntlich bei Verwendung starker Reduktionsmittel, wie Natrium ${ }^{8}$, Grignardverbindungen ${ }^{9}$, Aluminiumalkylverbindungen ${ }^{10}$ oder Ketylen ${ }^{11}$. Komplex gebundenes $\mathrm{CO}$ kann reduktiv in die Methylgruppe überführt werden, z. B. in der Umsetzung von $\left[\mathrm{CpMo}(\mathrm{CO})_{3}\left(\mathrm{P}_{3}\right)\right]^{+}$mit $\mathrm{NaBH}_{4}$ zu $\mathrm{CpMo}(\mathrm{CO})_{2}\left(\mathrm{P}_{3}\right) \mathrm{CH}_{3}{ }^{12}$. Die Reduktion von $\mathrm{CrCl}_{3} \cdot 3$ THF mit Kalium zu primär entstehendem aktiven Chrom und die erst dann einsetzende Bildung von $\mathrm{Cr}(\mathrm{CO})_{6}$ im CO-gefüllten Autoklaven ${ }^{13}$, die Beobachtungen Guainazzis bei der elektrochemischen, reduktiven Carbonylierung von $\mathrm{Cr}$ (III)Substraten ${ }^{14}$, sowie eigene Untersuchungen zur Darstellung von $\mathrm{Fe}(\mathrm{CO})_{5}$ durch anodische Auflösung von Eisen und anschließende reduktive Carbonylierung bei einer Zellspannung von $0,8-1,3 \mathrm{~V}$ in $\mathrm{CO}$ Atmosphäre legen andererseits eine Reaktion von 
Kohlenmonoxid mit einer Metallspezies erniedrigter Oxidationsstufe (z.B. 0, +1) zwingend nahe. Bei der Vielfalt von Untersuchungen von Umsetzungen des CO ist es erstaunlich, daß bis heute offenbar noch niemals ein Reduktionspotential im Sinne der Gleichung

$$
\mathrm{CO}+\mathrm{e} \rightarrow \mathrm{CO}^{-}
$$

angegeben wurde. Es erschien uns daher lohnend, danach zu suchen, da damit ein besseres Verständnis einer Reihe von Reaktionen des CO gegeben wäre.

\section{Experimentelles}

\subsection{Die Zelle und die elektronischen Geräte}

Die beschriebenen Messungen wurden mit Hilfe einer ungeteilten Dreischenkelzelle ausgeführt. Stets diente Platin als Gegenelektrode (eintauchende, wirksame Oberfläche $\left.c a \cdot 0,5 \mathrm{~cm}^{2}\right)$. Die eintauchende Oberfläche der Arbeitselektroden betrug etwa $0,3 \mathrm{~cm}^{2}$, der Abstand voneinander $3 \mathrm{~cm}$. Als Referenzelektrode wurde eine gesättigte Kalomelelektrode (SCE) eingesetzt. Bei allen Untersuchungen betrug die Durchlaufgeschwindigkeit $3000 \mathrm{mV} / \mathrm{min}$.

Eine Potentiostat Modell 68 FR 0,5 nach Wenking und ein Dreieckspannungsgenerator Wenking SMP 60 der Fa. G. Bank Elektronik, Göttingen, ermöglichten die Aufnahme der cyclovoltammetrischen Kurven.

\subsection{Elektroden}

Das verwendete Nickelnetz wurde mit Petroläther entfettet und zur Befreiung von Oxiden mit Essigsäure behandelt. Ni-, Co- und Ti-Bleche wurden durch Polieren mit feinem Schmirgelpapier gereinigt.

\subsection{Elektrolyt}

In allen Untersuchungen wurde eine gesättigte Lösung von $\mathrm{LiClO}_{4}$ in absolutem, argongesättigtem Dimethoxy-äthan, DME, als Elektrolyt verwendet.

\subsection{Kohlenmonoxid}

Das Gas* wurde keiner speziellen Reinigung unterworfen. Im Massenspektrum zeigten sich geringe Verunreinigungen durch $\mathrm{O}_{2}, \mathrm{CO}_{2}$ und $\mathrm{Ar}$ jedoch keine mit höheren Massenzahlen. Diesen minimalen Verunreinigungen wurde dadurch Rechnung getragen, daß eine $\mathrm{CO}_{2^{-}}$und eine $\mathrm{O}_{2}$-gesättigte Elektrolyt-Lösung mit in die Meßreihen aufgenommen und gezeigt wurde, daß die cyclovoltammetrischen Kurven sich dadurch im Vergleich zum Argesättigten Elektrolyten nicht maßgeblich veränderten.

* Der BASF-AG danken wir für die Überlassung des CO-Gases.

\subsection{Versuchsergebnisse}

2.5.1. Cyclovoltammetrische Untersuchungen zur Reduktion von $\mathrm{CO}$ an einer Nickelkathode im Bereich von 0 bis $-3,0 \mathrm{~V}$ gegen SCE

In allen Cyclovoltagrammen unseres Elektrolytsystems trat an Nickelelektroden je ein kathodjscher Peak bei $-2,6 \mathrm{~V}$ und ein anodischer Peak bei $-1,9 \mathrm{~V}$ gegen SCE auf. Analog zur Lithiumabscheidung und -auflösung an einer Thalliumkatho$\mathrm{de}^{15}$ ordnen wir sie einer Legierungsbildung zwischen Lithium (aus dem Leitsalz) und Nickel bzw. der Lithiumoxidation zu. Nach 15 Zyklen bis zur jeweils beginnenden Lithiumabscheidung passivierten die Elektroden offenbar, so daß diese Peaks nicht mehr auftraten und auch später keinerlei Anzeichen für eine Umsetzung von CO erkennbar waren. Für unsere Messungen wurden daher stets frisch gereinigte Elektroden eingesetzt. Um Störungen durch gelöste Gase, vor allem $\mathrm{CO}_{2}$ oder $\mathrm{O}_{2}$, im verwendeten Kohlenmonoxid ausschließen zu können, wurden entsprechende Vorversuche angestellt. In Abb. 1

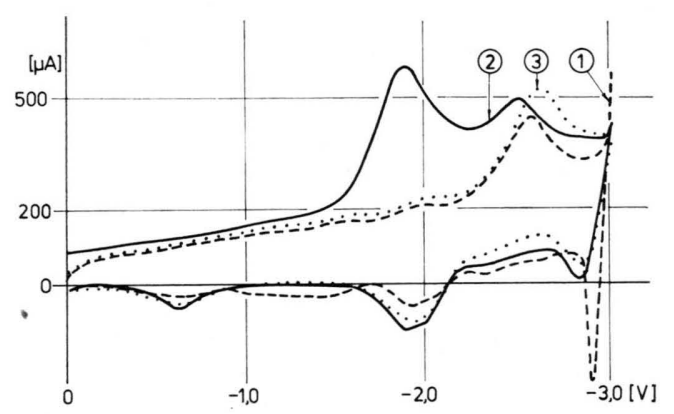

Abb. 1. Cyclovoltagramme an einer Nickelkathode: 1) einer gesättigten Lösung von $\mathrm{LiClO}_{4}$ in $\mathrm{DME}$ nach Durchleiten von $\mathrm{CO}_{2}$,

2) wie 1) mit zusätzlichem Durchleiten von CO, erster Zyklus.

3) wie 2) zweiter und folgende Zyklen.

ist das Cyclovoltagramm des Elektrolyten nach z.weiminütigem Einleiten von $\mathrm{CO}_{2}$ wiedergegeben. Nach gleichlangem Einleiten von $\mathrm{O}_{2}$ oder Argon wurden weitgehend ähnliche Kurven beobachtet. Nach anschließendem 2-minütigem Durchleiten von $\mathrm{CO}$ floß bei $-1,88 \mathrm{~V}$ deutlich ein kathodischer Strom, der mit der Adsorption von $\mathrm{CO}$ an der Elektrodenoberfläche verbunden ist. War die Nickelelektrode einmal vorher mechanisch gereinigt worden, so konnte der Peak der CO-Reduktion mit abnehmender Intensität in mehreren aufeinander folgenden Zyklen beobachtet werden. Dabei sank das Potential der CO-Reduktion von maximal $-2,05 \mathrm{~V}$ bei frisch polierten Elektroden auf $-1,88 \mathrm{~V}$ ab. Der Peak war dann wegen der durch Passivierung kleiner gewordenen wirksamen Oberfläche der $\mathrm{Ni}$-Kathode weniger breit ausgeprägt. Die Diffusion von $\mathrm{CO}$ an die Elektrodenoberfläche war nur wäh. 
rend eines schmäleren Potentialbereiches möglich. Spuren von $\mathrm{Ni}(\mathrm{CO})_{4}$ konnten den kathodischen Peak bei etwa $-1,9 \mathrm{~V}$ nicht hervorgerufen haben,

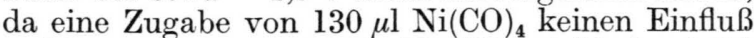
auf dessen Auftreten hatte. $\mathrm{Ni}(\mathrm{CO})_{4}$ wird bei $-2,9 \mathrm{~V}$ reduziert (bezogen auf $\left.\mathrm{Ag} / 10^{-3} \mathrm{M} \mathrm{AgClO}_{4}\right)^{16}$.

2.5.2. Cyclovoltammetrische Untersuchungen zur Reduktion von $\mathrm{CO}$ an einer Kobaltkathode im Bereich von 0 bis $-3,0 \mathrm{~V}$ gegen $\mathrm{SCE}$

Abb. 2 gestattet einen Vergleich des elektrochemischen Verhaltens von CO an einer Kobaltkathode mit dem $\mathrm{CO}_{2}$-gesättigten Grundelektrolyten. Ein Strommaximum wie im Falle der Nickelelektrode ist nicht zu erkennen, wohl aber ein deutlich ausgeprägter Stromanstieg ab $-2,4 \mathrm{~V}$, der in denjenigen der Lithiumabscheidung übergeht.

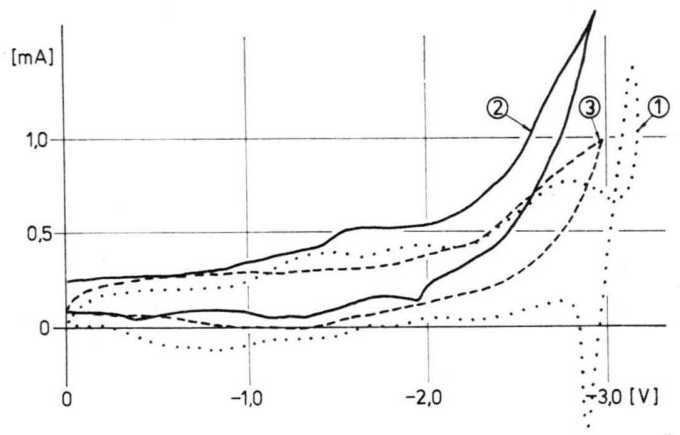

Abb. 2. Cyclovoltagramme an einer Kobaltkathode: 1) eines $\mathrm{CO}_{2}$-gesättigten Elektrolyten,

2) dritter Zyklus des CO-gesättigten Elektrolyten,

3) elfter Zyklus des CO-gesättigten Elektrolyten.

2.5.3. Cyclovoltammetrische Untersuchungen zur Reduktion von $\mathrm{CO}$ an einer Titankathode im Bereich von 0 bis $-3,0 \mathrm{~V}$ gegen $\mathrm{SCE}$

In Abb. 3 ist das elektrochemische Verhalten des argongesättigten Elektrolyten, des Elektrolyten

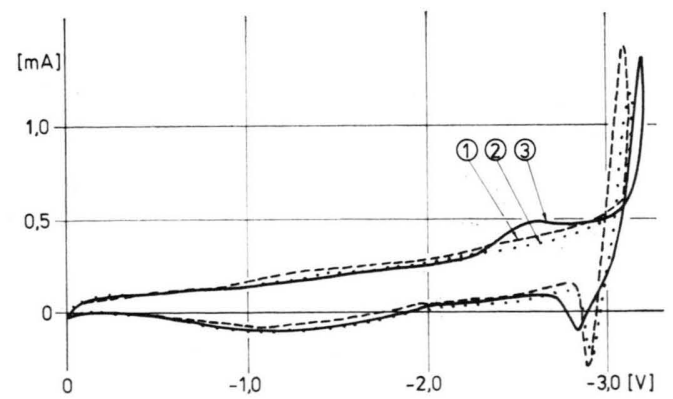

Abb. 3. Cyclovoltagramme an einer Titankathode:

1) eines argongesättigten Elektrolyten,

2) eines $\mathrm{CO}_{2}$ - und $\mathrm{O}_{2}$-gesättigten Elektrolyten,

3) eines $\mathrm{CO}$-gesättigten Elektrolyten. nach Einleiten von $\mathrm{CO}_{2}$ und $\mathrm{O}_{2}$ und des mit $\mathrm{CO}$ gesättigten Elektrolyten wiedergegeben. Ein Vergleich macht deutlich, daß bei $-2,6 \mathrm{~V}$ ein für anwesendes CO charakteristischer Strom seinen maximalen Wert erreicht hat.

Ein erneutes Einleiten von CO in den alten Elektrolyten bei Verwendung einer frisch polierten Arbeitselektrode ließ die Adsorption von $\mathrm{CO}$ noch deutlicher hervortreten, Abb. 4. Das Strommaximum konnte nach Durchlaufen des 2. Zyklus nach Einleiten von CO noch erkannt werden und veränderte sich von $-2,57$ nach $-2,46 \mathrm{~V}$.

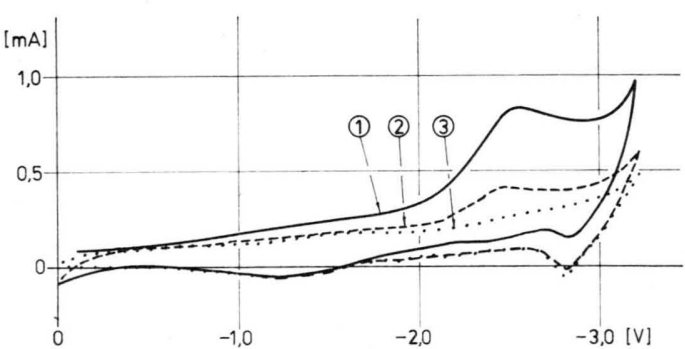

Abb. 4. Cyclovoltagramme an einer frisch polierten Titankathode; nach dem CO-Einleiten wurden drei Zyklen gemessen [1)-3)].

\section{Diskussion}

Die Untersuchungen ergaben, daß die irreversible Reduktion von Kohlenmonoxid offensichtlich nur nach vorangehender Chemisorption oder chemischer Bindung an der Elektrodenoberfläche beobachtet werden kann.

Die Adsorption von CO an Metallen oder Metalloxiden war Gegenstand vieler Arbeiten ${ }^{17-20}$. In diesem Zusammenhang sei auf Extended-HückelModellbetrachtungen der Wechselwirkung von Kohlenmonoxid mit Übergangsmetallen hingewiesen $^{21}$. Im Falle von Nickel werden dabei zwei Chemisorptionszustände diskutiert. Eine trigonale Struktur, in der $\mathrm{CO}$ an zwei Nickelatome gebunden ist, wird einer geringen $\mathrm{CO}$-Belegung zugeordnet, ansonsten wird eine lineare Struktur postuliert.

Dabei sind die Energieminima wesentlich stärker ausgeprägt als beispielsweise bei Kupfer.

Messungen der Oberflächenpotentiale von Nickel und Platin vor und nach Adsorption von Kohlenmonoxid führen zur Erkenntnis, daß die Belegung der Oberfläche im Falle des Nickels einen größeren Einfluß auf deren Änderung hat; sie weisen somit auf eine stärkere Chemisorption an diesem Übergangsmetall hin ${ }^{22}$. Diese Beobachtungen stehen in Übereinstimmung mit unserem Befund der unter. 
schiedlichen Reduktionspotentiale an verschiedenen Metallen.

Cyclovoltammetrische Untersuchungen mit Graphit als Kathodenmaterial und dem beschriebenen Elektrolyten ergaben im zugänglichen Bereich von 0 bis $-1,7 \mathrm{~V}$ gegen SCE keinen CO-spezifischen Reduktionspeak. Ab diesem Potential erfolgt der Einbau von Lithium in das Graphitgitter ${ }^{23}$ und eine Reduktion des $\mathrm{CO}$ an Graphit mißlingt.

Zusammenfassend läßt sich also sagen, daß Kohlenmonoxid an Nickel-, Kobalt- und Titankathoden irreversibel reduziert werden kann. Eine ausgeprägte Potentialabhängigkeit von dem jeweiligen Kathodenmaterial spricht für Chemisorption des Kohlenmonoxids vor der elektrochemischen Reduktion und vermutlich auch bei allen seinen Reduktionsreaktionen mit Metallen oder geeigneten Metallverbindungen. Bei reduzierenden Umsetzungen von $\mathrm{CO}$ ist durch geeignete Wahl des Elektrodenmaterials eine Absenkung des erforderlichen Reduktionspotentials in einen für die praktische An-

${ }^{1}$ I. Mitt.: siehe H. P. Fritz und Th. WÜrminghaUSEN, J. Electroanal. Chem. 54, 181 [1974].

2 A. Joannis, Compt. Rend. 116, 1518 [1894]; 158, 874 [1914].

3 J. Liebig, Ann. Chem. 11, 182 [1834].

${ }^{4}$ E. Weiss und W. Büchner, Helv. Chim. Acta 46, 1121 [1963].

5 W. Büchner, Helv. Chim. Acta 49, 907 [1966].

${ }^{6}$ O. Prziza, Dissertation TH Berlin, 1913.

7 G. Silvestri, S. Gambino, G. Fillardo, M. GuaiNAzzI und R. Ercoli, Gazz. Chim. Ital. 102, 818 [1972].

8 H. E. Podall, H. B. Prestridge und H. Shapiro, J. Amer. Chem. Soc. 83, 2057 [1961].

9 A. Job und A. Cassal, Compt. Rend. 183, 392 [1926].

10 L. J. Zakharkin, V. V. Gavrilenko und O. Yu. Olehlobystin, Isvest. Akad. Nauk. SSSR, Otdel. Khim. Nauk. 1958, 100.

${ }^{11}$ R. D. Closson, L. R. Buzbee und G. C. Ecke, J. Amer. Chem. Soc. 80, 6167 [1958]. wendung durchaus praktikablen Bereich möglich, wie es die Elektrosynthese der Quadratsäure ${ }^{7}$ zeigt. Der Mechanismus läßt sich etwa nach folgendem Schema darstellen, Abb. 5, der davon ausgeht, daß vier CO-Moleküle auf der Elektrodenoberfläche adsorbiert, reduziert und in Form der aus der Literatur bekannten Spezies desorbiert werden.

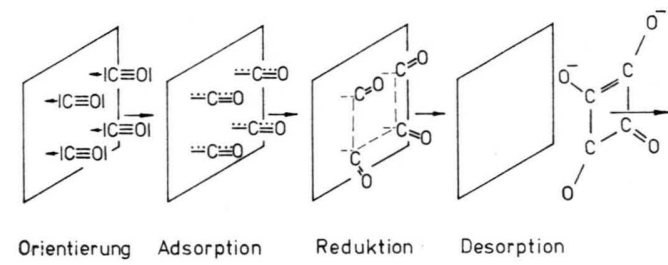

Abb. 5. Reaktionsschema der Quadratsäure-Bildung an einer Kathodenoberfläche.

Wie danken der Deutschen Forschungsgemeinschaft, Bonn-Bad Godesberg, für die großzügige Förderung dieser Untersuchung.

12 P. M. Treichel und R. L. Shubkim, Inorg. Chem. 6, 1328 [1967].

13 R. D. Rieke, K. ÖFele und E. O. Fischer, J. Organometal. Chem. 76, C 19 [1974].

14 M. Guainazzi, G. Silvestri, S. Gambino und G. Filardo, J. Chem. Soc., Dal. 1972, 927.

15 J. O. Besenhard, Dissertation, TU München, 1973.

${ }^{16}$ R. E. Dessy, F. E. Stary, R. B. King und M. WaLDROP, J. Amer. Chem. Soc. 88, 471 [1966].

17 P. Stonehart, Elektrochim. Acta 18, 63 [1973].

18 H. C. YAO und M. Shelef, J. Chem. Phys. 78, 2490 [1974].

19 H. S. GaNdHI und M. Shelef, J. Catal. 24, 241 [1972].

20 K. Gossner und H. PölzL, Z. Phys. Chem. 90, 164 [1974].

21 H. Köllbel und K. D. Tillmetz, Ber. d. Bunsenges. 76,1157 [1972].

22 G. J. H. Dorgelo und W. M. H. SAChtLer, Naturwissenschaften 46, 576 [1959].

23 J. O. Besenhard und H. P. Fritz, J. Electroanal. Chem. 53, 329 [1974]. 